\title{
Tapered optical fiber for Micro-Newton force sensor
}

\author{
Adrien Godet, Jacques Chrétien, Kien Phan Huy, Jean-Charles Beugnot \\ Institut FEMTO-ST, UMR 6174 CNRS, Université Bourgogne Franche-Comté, Besançon, France
}

\begin{abstract}
We used Brillouin scattering in silica nanofiber to demonstrate a microNewton force sensor having weak optical losses and using only one access of the nanofiber. The measurements are in good agreement with the theoretical model.
\end{abstract}

Fiber force sensor, offering both a broad detection range and a high sensitivity, is essential for several applications from earthquake monitoring to automation system [1]. Due to the small transverse dimension, the tapered optical fiber have a number of optical and mechanical properties that make them very attractive for both fundamental physics and technological applications [2-5]. Contrary to standard telecom fiber where the Brillouin scattering effect is characterized by a single Lorentzian resonance centred at 10.86 GHz (@1550nm), in tapered silica fiber, we identified several Brillouin resonances at different frequencies from $5 \mathrm{GHz}$ to $10 \mathrm{GHz}$ coming from surface, shear and compression elastic waves [6]. In this work, we used the high sensitivity of Brillouin scattering to longitudinal strain and the nanoscale cross-section of nanofiber (the waist part of the tapered fiber) in order to design a very sensitive force at the $10^{-6}$ Newton range. We report a point fibre force sensor with sensitivity up to $0.66 \mu \mathrm{Newton} / \mathrm{MHz}$ by using a nanofiber diameter of 630 $\mathrm{nm}$.

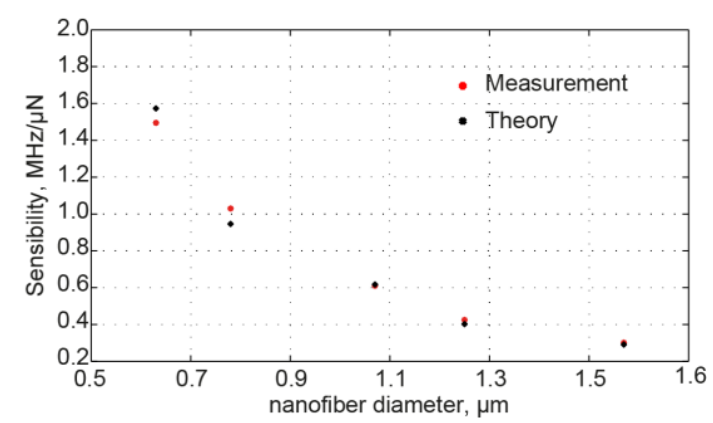

Fig. 1. Brillouin sensitivity as a function of the nanofiber diameter, experiment (red) and theory (black).

The tapered optical fibers are produced from commercial optical fibers using well know heat-brush technique. The geometrical parameter of tapered optical fiber as nanofiber diameter and homogeneity are estimated by Brillouin spectroscopy [8]. We used a commercial strain gauge which is able to measure forces in the 10-3 Newton range. One end of the taper is fixed to the translation stage while the other end is fixed to the force sensor. After benchmarking of force sensor, we moved the translation stage by step of $100 \mu \mathrm{m}$ and measured the spontaneous backscattering Brillouin spectrum using heterodyne detection.

In figure 1, the experimental results of BFS sensitivity as a function of nanofiber diameter are represented. The analytical model [8] is in good agreement with experimental measurement.

To conclude, we have experimentally investigated the force sensing potential of Brillouin scattering in tapered silica optical fiber. The linear dependence of Brillouin resonance from compression elastic wave is in good agreement with analytical model. Sensibility (1 $\mathrm{MHz} / \mu \mathrm{N})$ and dynamic ratio (1:2000) can be tune with diameter of taper and the total losses is not modified and below $0.1 \mathrm{~dB}$. These results demonstrate the strong potential of tapered optical fiber for vibrational acoustic sensor.

The authors would like to acknowledge the financial support of Agence Nationale de la Recherche (FUNFILM-ANR-16-CE24-0010-03).

\section{References}

1. N. J. Lindsey, T. D. Craig and J. B. Ajo-Franklin "Illuminating seafloor faults and ocean dynamics with dark fiber distributed acoustic sensing," Science 366, 1103-1107 (2019).

2. W. Luo, J.-L. Kou, Y. X. Chen, F. Xu, and Y. Lu. "Ultra-highly sensitive surface-corrugated microfiber Bragg grating force sensor," Applied Physics Letters 101, 133502 (2012).

3. T. Wieduwilt, S. Brückner and H. Bartelt., "High force measurement sensitivity with fiber Bragg gratings fabricated in uniform-waist fiber tapers," Meas. Sci. Technol. 22075201 (2011).

4. Y. Chen, S-C. Yan, X. Zheng, F. Xu, and Y.-Q. Lu, "A miniature reflective micro-force sensor based on a microfiber coupler," Optics Express 22, 2443-2450 (2014).

\footnotetext{
* Corresponding author: adrien.godet $@$,femto-st.fr
} 
5. L. Tong, "Micro/Nanofibre Optical Sensors: Challenges and Prospects," Sensors 18, 903, (2018).

6. J-C. Beugnot, S. Lebrun, G. Pauliat, H. Maillotte, V. Laude, and T. Sylvestre, "Brillouin light scattering from surface acoustic waves in a subwavelengthdiameter optical fibre," Nat. Commun. 5, 5242 (2014).

7. A Godet, T Sylvestre, V Pécheur, J Chrétien, J-C. Beugnot, and K. P. Huy., "Nonlinear elasticity of silica nanofiber," APL Photonics 4, 080804 (2019).

8. A. Godet, A. Ndao, T. Sylvestre, V. Pecheur, S. Lebrun, G. Pauliat, J-C. Beugnot, and K. P. Huy," Brillouin spectroscopy of optical microfibers and nanofiber," Optica 4, 1232-1238 (2017). 